\title{
3D OBJECT RECONSTRUCTION FROM SWISSRANGER SENSOR DATA USING A SPRING-MASS MODEL
}

\author{
Babette Dellen ${ }^{1,2}$, Guillem Alenyà ${ }^{2}$, Sergi Foix ${ }^{2}$, and Carme Torras ${ }^{2}$ \\ ${ }^{1}$ Bernstein Center for Computational Neuroscience, Max-Planck Institute for Dynamics and Self-Organization, \\ Bunsenstrasse 10, Göttingen, Germany \\ ${ }^{2}$ Institut de Robòtica i Informàtica Industrial (CSIC-UPC), Llorens i Artigas 4-6, 08028 Barcelona, Spain \\ bkdellen@bccn-goettingen.de,galenya@iri.upc.edu,sfoix@iri.upc.edu,torras@iri.upc.edu
}

Keywords: $\quad$ Swissranger Sensor, 3D Reconstruction, Spring-Mass Model.

\begin{abstract}
We register close-range depth images of objects using a Swissranger sensor and apply a spring-mass model for $3 \mathrm{D}$ object reconstruction. The Swissranger sensor delivers depth images in real time which have, compared with other types of sensors, such as laser scanners, a lower resolution and are afflicted with larger uncertainties. To reduce noise and remove outliers in the data, we treat the point cloud as a system of interacting masses connected via elastic forces. We investigate two models, one with and one without a surface-topology preserving interaction strength. The algorithm is applied to synthetic and real Swissranger sensor data, demonstrating the feasibility of the approach. This method represents a preliminary step before fitting higher-level surface descriptors to the data, which will be required to define object-action complexes (OACS) for robot applications.
\end{abstract}

\section{INTRODUCTION}

The automatic reconstruction and model building of complex physical objects is important to guide robotic tasks such as grasping and view-point changes, and more in general to predict the outcome of robot manipulations. Due to improvements during the last decade in the field of 3D time-of-flight sensors (TOF), faster and more accurate data can now be obtained from these sensors. The Swissranger sensor provides an excellent tool for 3D robot exploration tasks. The camera can be mounted on a robot arm, depth images can be acquired in real time, and objects can be simultaneously manipulated and reconstructed. The resulting depth images in this specific application are of low resolution and contain large uncertainties, requiring the adaptation and development of suitable models.

Previously, several methods have been proposed to reconstruct 3D object surfaces from a cloud of 3D data points, e.g. dynamic particles [Szeliski et al., 1992], implicit-surface based methods [Hoppe et al., 1992], volumetric methods [Curless and Levoy, 1996], tensor-voting based methods [Tang and Medioni, 2002], Voronoi-based surface reconstruc- tion [Amenta et al., 1998], level sets [Zhao et al., 2000], and surface fitting with radial basis functions [Carr et al., 2001]. Techniques based on dynamic particles [Szeliski et al., 1992] have the advantage that a microscopic model of the object is constructed, which can be used to model outcome of robot action applied to deformable objects, e.g. the manipulation of a table cloth [Cuen et al., 2008]. In this paper, we propose a method based on a system of elastically interacting masses. The proposed model differs from previous approches based on dynamic particles in the way the data is incorporated into the model and in the specific choices of the interaction functions. A novel feature of this algorithm is the inclusion of a damping and a noise term which drive the system towards a local minimum, an idea similar to simulated annealing. The model constitutes a preliminary step before fitting higher-level surface descriptors to the data. These entities will then provide a solid basis for guiding object actions, i.e. viewpoint changes and actions of the robot gripper.

The reconstruction process consists of the following steps: Image capture (a), coarse registration (b), fine registration (c), and implicit surface modeling via a spring-mass model (d), which is introduced in Sec- 
tion 2. Steps a-c are described in Section 3.

\section{THE MODEL SYSTEM}

The basic framework we consider can be defined as follows. Let $P$ be a set of data points with measured position $\mathbf{X}_{\mathbf{p}}=\left(X_{p}, Y_{p}, Z_{p}\right)$ in a three-dimensional space. To each data point, we assign a mass point defined by a continuous position variable $\mathbf{x}_{\mathbf{p}}=$ $\left(x_{p}, y_{p}, z_{p}\right)$ and a velocity variable $\mathbf{v}_{\mathbf{p}}=\left(u_{p}, v_{p}, w_{p}\right)$.

The masses are moving under the influence of a data force

$$
F_{\text {data }}=k\left(\mathbf{X}_{\mathbf{p}}-\mathbf{x}_{\mathbf{p}}\right),
$$

where $k$ is a parameter.

Each mass $q$ exerts a force

$$
F_{\text {int }}(p, q)=\kappa(p, q)\left(\mathbf{x}_{\mathbf{q}}-\mathbf{x}_{\mathbf{p}}\right)
$$

on $p$, if $q$ is in the neighborhood $N(p)$ of $p$.

We consider two different models, $\mathrm{A}$ and $\mathrm{B}$. In model $\mathrm{A}, \kappa(p, q)$ is equal to a constant $c$, while in model $\mathrm{B}$ the function $\kappa(p, q)$ depends on the angle between the surface normal of particle $q$ and the difference vector $\mathbf{x}_{\mathbf{p}}-\mathbf{x}_{\mathbf{q}}$, such that

$$
\kappa(p, q)=2 c\left[\pi / 2-\theta\left(\mathbf{x}_{\mathbf{p}}-\mathbf{x}_{\mathbf{q}}, \mathbf{n}_{\mathbf{q}}\right)\right] / \pi,
$$

where $\mathbf{n}_{\mathbf{q}}$ is the surface normal at $q$ and

$$
\theta(\mathbf{a}, \mathbf{b})= \begin{cases}\angle(\mathbf{a}, \mathbf{b}) & \text { if } \angle(\mathbf{a}, \mathbf{b}) \leqq \pi / 2 \\ \pi-\angle(\mathbf{a}, \mathbf{b}) & \text { if } \angle(\mathbf{a}, \mathbf{b})>\pi / 2\end{cases}
$$

Note that $\angle(\mathbf{a}, \mathbf{b})$ is the inner angle enclosed by a and b and thus does not grow larger than $\pi$. Eq. 4 ensures that the interaction force depends only on the orientation of the surface normal relative to the mass.

A schematic comparison of model A and B is provided in Fig. 1. While the interaction forces in model A only depend on the relative distances of neighboring particles, model B takes into account the position of a particle with respect to the surface orientation of the neighboring particles. This has the advantage that the data is preferably smoothed in the direction of the local surface normal, thus reducing undesired contractions of the object model and oversmoothing at edge discontinuities.

The dynamics of the system is described by a system of coupled ordinary differential equations

$$
\begin{aligned}
& d \mathbf{x}_{\mathbf{p}} / d t=\mathbf{v}_{\mathbf{p}} \\
& d \mathbf{v}_{\mathbf{p}} / d t=F_{\text {data }}+F_{\text {int }}+\tau-\gamma \mathbf{v}_{\mathbf{p}},
\end{aligned}
$$

where $\tau$ is a noise term and $\gamma_{\mathbf{p}}$ a damping term with damping constant $\gamma$. These additional forces have been added to move the dynamical system towards a local minimum.

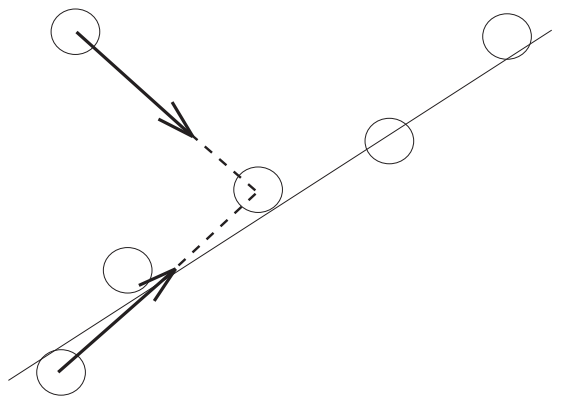

(a) Model A

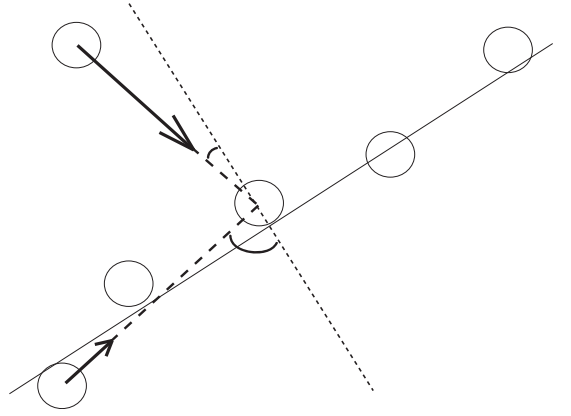

(b) Model B

Figure 1: Schematic of particle interaction models. (a) In model A, the interaction forces (thick arrow) are independent of the orientation of the local surface (thin line). (b) In model $\mathrm{B}$, the interaction forces are stronger in the direction of the local surface normal

\subsection{Finding a Local Minimum}

The system of differential equations is solved using a 4th order Runge Kutta technique with a step size of 0.1 , starting from random initial conditions. Cooling is introduced through the damping force and the noise term $\tau$. With the course of time, we decrease the noise according to

$$
\tau=p_{r}\left(n_{i}-t\right) / n_{i}
$$

where $n_{i}$ is the total number of iterations and $t$ is the current iteration number. The number $p_{r}$ is drawn from a Gaussian distribution with a standard deviation of 5 pixels.

\subsection{Surface Normals}

Following Liang and Todhunter [Liang and Todhunter, 1990], we define the local covariance matrix at $p$ as

$$
C=\sum_{q \in N_{s}(p)}\left(\mathbf{x}_{\mathbf{q}}-\mathbf{x}_{\mathbf{m}}\right) \cdot\left(\mathbf{x}_{\mathbf{q}}-\mathbf{x}_{\mathbf{m}}\right)^{T} / n
$$


where $n$ is the number of points in the local neighbor$\operatorname{hood} N_{s}(p)$ of $p$, and

$$
\mathbf{x}_{\mathbf{m}}=\sum_{q \in N_{s}(p)} \mathbf{x}_{\mathbf{q}} / n
$$

is the mean position vector. The local plane which minimizes, in the least squares sense, the orthogonal projections of all points in $N_{s}(p)$ onto the plane is given by the two eigenvectors corresponding to the two largest eigenvalues.

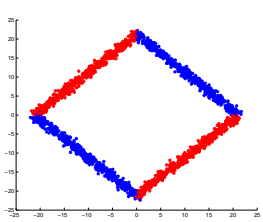

(a) Data view 1

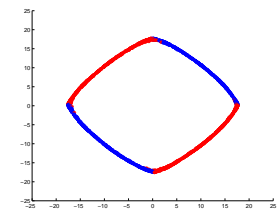

(c) Model A view 1

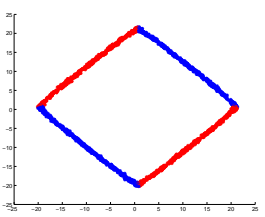

(e) Model B view 1

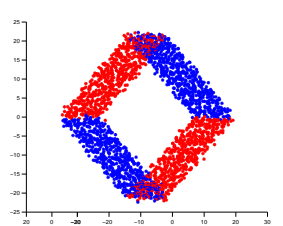

(b) Data view 2

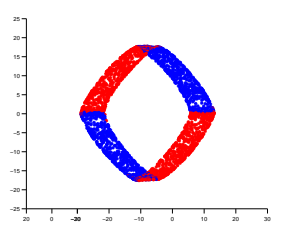

(d) Model A view 1

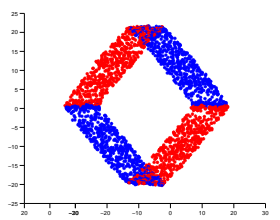

(f) Model B view 2
Figure 2: (a-b) Original data, seen from two different views. (c-d) Results model A, seen from two different views. (e-f) Results model B, seen from two different views.

\section{IMAGE REGISTRATION}

For our experiments a Swissranger SR3100 camera has been used. This new type of sensor has the ability of simultaneously measuring depth and intensity for every pixel in the image. The camera is provided with its own illumination system, composed by a set of modulated infra-red LEDs. The sensor has a low pixel resolution of $176 \times 144$ but a high frame rate average of $25 \mathrm{fps}$. This high frame rate makes the Swissranger camera a suitable sensor for real time applications.

Depth images of the object are taken for different views of the scene. Coarse global registration is achieved via coarse pose estimation using multiple correspondences of invariant geometric features extracted from the 3D point clouds [Chua and Jarvis, 1997]. After the coarse pose registration, an iterative closest point algorithm (ICP) is applied in order to achieve a fine pose registration [Besl and McKay, 1992].

\subsection{TOF Errors and Integration Time}

The 3D-Range images captured with TOF sensors contain different systematic errors that falsify depth measurements [Oprisescu et al., 2007]. Systematic errors due to environmental electromagnetic signals such as sunlight and auto-lighting reflections have been minimised by restricting the environmental scene to a suitable non-reflecting uniform textured one. After recalibration by the manufacturer and focus distance tuning, short range image extraction has proved to be appropriate. As a consequence, the intrinsic errors due to oversaturation have been considered as negligible.

One of the TOF sensor parameters that allows to obtain close range pictures is Integration Time. This parameter has to be very low in order to assure good readings from the camera at a range of $30 \mathrm{~cm}$. However, it adds depth variance into the pixels of the image. In order to deal with this problem the average of 15 3D-Range images is computed. In order to enhance the quality of the images, depth discontinuities due to jump edge surfaces are filtered using the method proposed by [Fuchs and May, 2008].

In the constrained environment of our set-up, the object is segmented from the background using a depth threshold.

\subsection{Invariant Geometric Feature Extraction and Rigid Registration}

Different invariant geometric feature extraction and rigid registration methods have been studied and used in the literature [Seeger and Laboureux, 2000]. We use a method proposed by Chua and Jarvis (1997). This method defines rotation and translation invariant features called "Point Signatures" for every point of a free-form surface with the benefit that it does not need partial derivative computations. This approach has been chosen due to its simplicity and fast behaviour.

\section{RESULTS}

For parameter choices $\gamma=0.5, k_{n}=30, k=1, c=$ $20 / k_{n}$, and $n_{i}=1000$, we compute the results of 
model A and model B for a synthetic example. Model $B$ is then applied to the Swissranger sensor data. The data was scaled by a factor 0.04 before application of the model, and returned to its original scale afterwards.

Each aquired data point is assigned a mass point in the model. We find all $k_{n}$ nearest neighbors defining the neighborhood $N(p)$ of each mass $p$. The surface normals are computed by finding $k_{s}=12$ nearest neighbors. The neighbors are not altered during the simulation of the dynamical system.

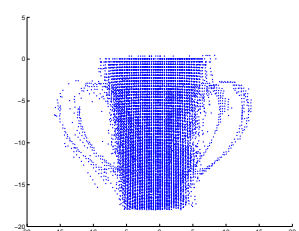

(a) Raw data

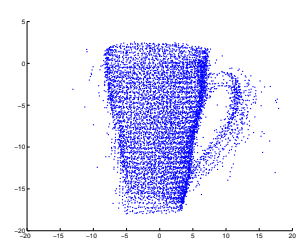

(c) Fine registration

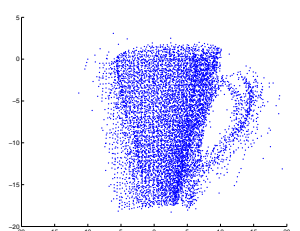

(b) Coarse registration

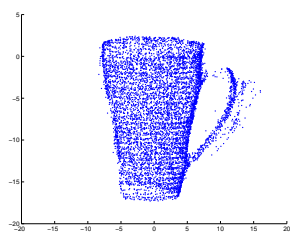

(d) Result model B
Figure 3: Modeling process of Swissranger data taken from a mug. (a) Raw data with superimposed unmerged views. (b) Coarse registration. (c) Fine registration. (d) Final result using model $\mathrm{B}$.

As a synthetic test example, we choose an object in the shape of an open box, which is then acquired from two different view points, separated by an angle $\pi$, via an orthographic projection. We further assume that the data acquisition process is noisy. In the data space, the object is represented by a noisy point cloud. Two different views of this point cloud are shown in Fig. 2 (a-b). For illustration purposes, we mark all data points with a surface normal closer to a vector pointing in the upper left corner as red, and all other data point as blue. We simulate the dynamical system of model A for this synthetic example. Two views of the model data, corresponding to the views shown for the raw data, are depicted in Fig. 2(c-d). The model data is much smoother than the original data. However, since the interaction forces do not take into account the topology of the object, oversmoothing is observed at edge discontinuities. Further, the object model is smaller than the original object. In Fig. 2(ef), the result of model B are depicted. Unlike in model $\mathrm{A}$, the modeled object has the same size as the original object, and edge discontinuities have been preserved during the smoothing process.

We apply model B to the depth images of a ceramic mug acquired with the Swissranger sensor. The different stages of the modeling process, i.e. raw data acquisition, coarse and fine registration, and results of the subsequent application of the spring-mass model, are shown in Fig. 3, a-d, respectively. At the end of the process, the mug model has a clear shape and most of the outliers have been removed, except for the handle. In Fig. 4, the color-coded orientations of local surface patches for the fine registration and the final result after applying model B are depicted. Surfaces of mug model B are smoother than the surfaces at the fine registration stage, thus providing a good basis for subsequent applications.

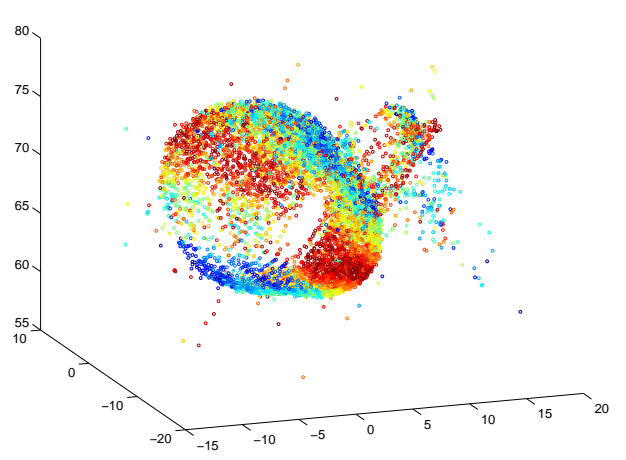

(a) Fine registration

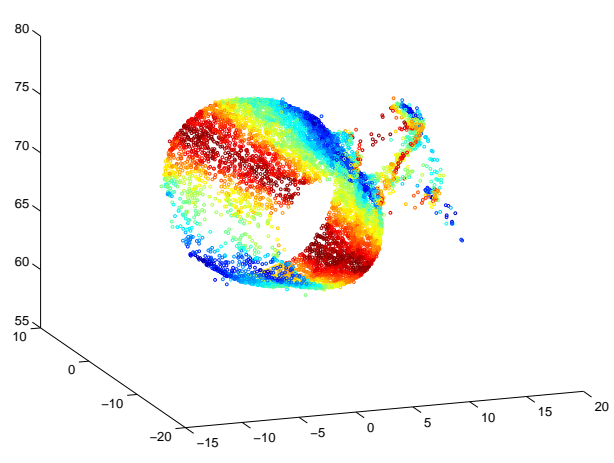

(b) Result model B

Figure 4: Color-coded orientation of local surface patches for the mug result (a) Fine registration. (b) Final result of model B. 


\section{CONCLUSIONS}

\subsection{Summary}

Within the larger context of a robot manipulation task, we proposed a 3D model for the reconstruction of 3D objects from Swissranger sensor data. Depth images are captured with a Swissranger sensor. After taking depth images from different views, the images are merged via a coarse registration method [Chua and Jarvis, 1997]. The shape of the 3D point cloud is enhanced through application of an iterative closest point algorithm [Besl and McKay, 1992]. The resulting data is of low resolution and afflicted with large uncertainties. Modeling the 3D point cloud as a system of dynamic masses interacting via springlike elastic forces reduces these deficiencies. Since the interaction between masses is dependent on the orientation of local surfaces fitted to the mass points, noise and outliers are removed without causing oversmoothing at edge discontinuities.

\subsection{Future Work}

Our long-term goal is to model 3D objects during the execution of a robot task. The proposed model constitutes a preliminary step before fitting higherlevel surface descriptors which may then be used for view planning or action selection. The spring-mass model further provides a framework for modeling deformable objects. The outcome of actions can be predicted by adding an external force to the dynamical system of the object, e.g. representing a robot gripper.

\section{ACKNOWLEDGEMENTS}

This work has received support from the BMBF funded BCCN Göttingen, the EU Project PACOPLUS under contract FPG-2004-IST-4-027657, and the Generalitat de Catalunya through the Robotics group. G. Alenyà was supported by the CSIC under a Jae-Doc Fellowship.

\section{REFERENCES}

Amenta, N., Bern, M., and Kamvysselis, M. (1998). A new voronoi-based surface reconstruction algorithm. In SIGGRAPH '98, pages 415-421, New York, NY, USA. ACM.
Besl, P. J. and McKay, N. D. (1992). A method for registration of 3-d shapes. IEEE Transactions on Pattern Analysis and Machine Intelligence, 14(2):239-256.

Carr, J., Beatson, R., Cherrie, J., Mitchell, T., Fright, W., McCallum, B., and Evans, T. (2001). Reconstruction and representation of $3 \mathrm{~d}$ objects with radial basis functions. In Fuime, E., editor, Proceedings of SIGGRAPH 2001, pages 67-76. ACM Press/ACM SIGGRAPH.

Chua, C. S. and Jarvis, R. (1997). Point signatures: a new representation for $3 \mathrm{~d}$ object recognition. International Journal of Computer Vision, 25(1):63-85.

Cuen, S., Andrade, J., and Torras, C. (2008). Action selection for robotic manipulation of deformable objects. In Proc. ESF-JSPS Conference on Experimental Cognitive Robotics, Kanagawa.

Curless, B. and Levoy, M. (1996). A volumetric method for building complex models from range images. In Proceedings of SIGGRAPH'96.

Fuchs, S. and May, S. (2008). 3d pose estimation and mapping with time-of-flight cameras. In Workshop on $3 D$ Mapping, IEEE International Conference on Intelligent Robots and Systems (IROS 2008).

Hoppe, H., DeRose, T., Duchamp, T., McDonald, J., and Stuetzle, W. (1992). Surface reconstruction from unorganized points. In SIGGRAPH '92, pages 71-78, New York, NY, USA. ACM.

Liang, P. and Todhunter, J. S. (1990). Representation and recognition of surface shapes in range images. Computer Vision, Graphics and Image Procesing, 52(10):78-109.

Oprisescu, S., Falie, D., Ciuc, M., and Buzuloiu, V. (2007). Measurements with tof cameras and their necessary corrections. In Proc. ISSCS, pages 13-14.

Seeger, S. and Laboureux, X. (2000). Feature extraction and registration: An overview. In Girod, B., Greiner, G., and Niemann, H., editors, Principles of $3 \mathrm{D} \mathrm{Im}$ age Analysis and Synthesis, pages 153-166, BostonDordrecht-London. Kluwer Academic Publishers.

Szeliski, R., Tonnesen, D., and Terzopoulos, D. (1992). Modeling surfaces of arbitrary topology with dynamic particles. CVPR'93, pages 82-87.

Tang, C. and Medioni, G. (2002). Curvature-augmented tensor voting for shape inference from noisy $3 \mathrm{~d}$ data. IEEE Transactions on Pattern Analysis and Machine Intelligence, 26(6):858-864.

Zhao, H., Osher, S., Merriman, B., and Kang, M. (2000). Implicit and non-parametric shape reconstruction from unorganized data using a variational level set method. In Proceedings of CVIU. 